\title{
NECESSARY CONDITIONS IN THE PROBLEMS OF MAYER IN THE CALCULUS OF VARIATIONS*
}

BY

\author{
GILLIE A. LAREW
}

\section{INTRODUCTION}

The problem usually called in the Calculus of Variations the "Problem of Mayer" may be formulated as follows: $\dagger$

Among all systems of functions, $y_{0}(x), y_{1}(x), \cdots, y_{n}(x)$, which satisfy the $m+1$ differential equations

$$
\phi_{a}\left(x ; y_{0}, \cdots, y_{n}, y_{0}^{\prime}, \cdots, y_{n}^{\prime}\right)=0 \quad(\alpha=0,1, \cdots, m ; m<n)
$$

and for which $y_{0}, \cdots, y_{n}$ take on fixed values $y_{01}, \cdots, y_{n 1}$ at $x=x_{1}$ while $y_{1}, \cdots, y_{n}$ take on fixed values $y_{12}, \cdots, y_{n 2}$ at $x=x_{2}$, it is required to determine a system giving $y_{0}(x)$ a minimum or a maximum at $x_{2}$.

The deduction of the Euler-Lagrange differential equations as a first necessary condition has been closely studied, $\ddagger$ and Mayer in his paper in the Leipziger B e r c h t e of 1878, where the problem stated above was first formulated, shows that there are necessary for a minimum conditions analogous to those of Clebsch and Jacobi for other problems of the Calculus of Variations. Kneser has also discussed the sufficient conditions for a minimum and the general definition of a field of extremals, both in his Lehrbuch and in a recent paper. $\S$ Little subsequent attention, however, has been paid to the necessary conditions of Clebsch and Jacobi or to a necessary condition analogous to that of Weierstrass.

It is the purpose of this paper to inquire systematically into the question of necessary conditions. In investigating the corner-point condition for socalled "discontinuous solutions" the theorem on the necessary condition of Euler is extended to include arcs which are continuous, but which may have a finite number of corners. A formulation and proof is supplied for the neces-

* Presented to the Society, Oct. 26, 1918.

$\dagger$ Cf. Bolza, Vorlesungen über Variationsrechnung, p. 573.

$\ddagger$ For references see Bolza, loc. cit.; also Hadamard, Leçons sur le Calcul des Variations, Chap. VI, p. 223, ff.

$\S$ Lehrbuch der Variationsrechnung, Abschnitt VII, §§59, 60; Archiv der Mathematik und Physik, vol. 24 (1915), p. 26. 
sary condition of Weierstrass, and the Jacobi condition is deduced in much more simple fashion than usual by an application of the Euler equations and the corner-point condition to the second variation. The problem of the minimum is considered throughout, the changes required in the case of a maximum being obvious.

\section{Preliminary Considerations}

For convenience, the letters $i, j, \alpha, \beta, p, \mu, r, s, \sigma$, will be used as indices with the ranges indicated in the following table:

$$
\begin{aligned}
i, j=0,1,2, \cdots, n ; & p=1,2, \cdots, n ; \\
\alpha, \beta=0,1,2, \cdots, m ; & \mu=1,2, \cdots, m ; \\
r, s=m+1, m+2, \cdots, n ; & \sigma=1,2, \cdots, 2 n+1 .
\end{aligned}
$$

The symbol $F$ will denote the Euler-Lagrange sum

$$
\begin{aligned}
F\left(x, y_{0}, y_{1}, \cdots, y_{n}, y_{0}^{\prime}, \cdots, y_{n}^{\prime}, \lambda_{0}, \cdots, \lambda_{m}\right) \\
\quad=\sum_{a} \lambda_{a} \phi_{a}\left(x, y_{0}, \cdots, y_{n}, y_{0}^{\prime}, \cdots, y_{n}^{\prime}\right) .
\end{aligned}
$$

The $\lambda$ 's appearing in this sum are the functions of $x$ sometimes called Lagrange multipliers. Their nature is explained in the theorems of $\S 3$. Partial derivatives will be indicated by subscripts as follows:

$$
\begin{gathered}
F_{i}=\partial F / \partial y_{i} ; \quad G_{i}=\partial F / \partial y_{i}^{\prime} ; \quad \phi_{a i}=\partial \phi_{a} / \partial y_{i} \\
\psi_{a i}=\partial \phi_{a} / \partial y_{i}^{\prime} ; \quad R_{i j}=\partial^{2} F / \partial y_{i}^{\prime} \partial y_{j}^{\prime} .
\end{gathered}
$$

The minimizing arc will be denoted by the symbol $E$. Its equations are

$$
y_{i}=e_{i}(x) \quad\left(x_{1} \leqq x \leqq x_{2}\right)
$$

and it is supposed to be of class $D^{\prime}$ * $^{*}$ The arguments of $E$ satisfy the differential equations (1) and the conditions $y_{i}\left(x_{1}\right)=y_{i 1}, y_{p}\left(x_{2}\right)=y_{p 2}$. The functions $\phi_{a}$ are of class $C^{\prime \prime \prime}$ in a neighborhood of $E$. A further hypothesis is that at no point on $E$ do all determinants of the matrix $\left\|\psi_{a i}\right\|$ vanish simultaneously. Some determinant including the column $\partial \phi_{a} / \partial y_{0}^{\prime}$ is supposed to be everywhere different from zero, and we may take it to be $\left|\psi_{a \beta}\right|$.

An admissible arc $y_{i}(x)$ is one of class $D^{\prime}$ which satisfies the differential equations $\phi_{a}=0$ and lies in a region of points $\left(x, y_{i}, y_{i}^{\prime}\right)$ in which the functions $\phi_{a}$ are of class $C^{\prime \prime \prime}$. A one-parameter family of such curves $y_{i}=Y_{i}(x, \epsilon)$ will be called an admissible family, if the function $Y_{i}(x, \epsilon)$ is continuous for all values $(x, \epsilon)$ near those defined by the conditions $x_{1} \leqq x \leqq x_{2}, \epsilon=0$,

* For the definition of "class" of a curve, see Bolza, loc. cit., pp. 13, 63. 
while $Y_{i}(x, \epsilon)$ and $Y_{i}^{\prime}(x, \epsilon)$ both have continuous derivatives with respect to $\epsilon$ in the same neighborhood, except possibly for a finite number of values of $x$. The minimizing arc $E$ is an admissible arc satisfying the end-conditions of the problem. If $E$ is imbedded in an admissible family, it will always be supposed to be contained in the family for the parameter value $\epsilon=0$.

The equations of variation for $E$ are the equations

$$
\sum_{i} \phi_{a i} \eta_{i}+\sum_{i} \psi_{a i} \eta_{i}^{\prime}=0,
$$

where the arguments of the derivatives $\phi_{a i}, \psi_{a i}$ are those of $E$. If the family $Y_{i}(x, \epsilon)$ is an admissible family containing $E$ for $\epsilon=0$, the equations of variation are satisfied by the derivatives $\eta_{i}=\left[\partial Y_{i} / \partial \epsilon\right]^{\epsilon=0}$, which are of class $D^{\prime}$. Every set of functions $\eta_{i}(x)$ of class $D^{\prime}$ which satisfies the equations of variation is called a set of admissible variations whether or not it arises as the set of derivatives mentioned, and irrespective. of the end-values assumed by the functions $\eta_{i}$.

The Euler-Lagrange differential equations for this problem are

$$
F_{i}-\frac{d}{d x} G_{i}=0 ; \quad \phi_{a}=0 .
$$

An $\operatorname{arc} E$ contains no singular point of these equations, if for every point of $E$ the determinant

$$
R\left(x, e_{i}, e_{i}^{\prime}, \lambda_{a}\right)=\left|\begin{array}{cc}
\partial G_{i} / \partial y_{j}^{\prime} & \psi_{a i} \\
\bar{\psi}_{a i} & 0
\end{array}\right| \neq 0,
$$

the matrix $\left\|\bar{\psi}_{a i}\right\|$ denoting the result of interchanging rows and columns in $\left\|\psi_{a i}\right\|$. A solution of the Euler-Lagrange equations is called an extremal. It is provable that the extremal $E$ can be imbedded in a family defined over $x_{1}-\delta<x<x_{2}+\delta$.

Bolza* has made a very inclusive generalization of the distinction between the two types of extremals called by Hahn "normal" and "anormal." The Mayer problem with fixed end-points is a special case of the problem considered by Bolza, and, for it, the definition of a normal extremal takes the following form: The arc $y_{i}=e_{i}(x)$ is normal on the interval $\left[x_{1} x_{2}\right]$ for the minimizing of $y_{0}\left(x_{2}\right)$, if there exist $2 n+2$ sets of admissible variations $\eta_{i}^{0}(x), \cdots, \eta_{i}^{(2 n+1)}(x)$ such that the matrix

$$
\left\|\begin{array}{ll}
\eta_{i}^{(j)}\left(x_{1}\right) & \eta_{i}^{(n+j+1)}\left(x_{1}\right)^{v} \\
\eta_{p}^{(j)}\left(x_{2}\right) & \eta_{p}^{(n+j+1)}\left(x_{2}\right)
\end{array}\right\|
$$

is of rank $2 n+1$. In the rows of this matrix $j$ varies, while $i$ and $p$ vary in the columns. As Bolza has noted, $\dagger$ it is easily proved that for a normal arc

$$
G_{0}\left(x_{2}\right)=\left[\partial F / \partial y_{0}^{\prime}\right]^{x=x_{2}} \neq 0 .
$$

* Mathematis che Annalen, vol. 74 (1913), p. 430.

† Loc. cit., p. 446. 
The extremals considered in this paper are supposed to be normal. As equations (2) are homogeneous in the functions $\lambda_{a}$, evidently, if $y_{i}=e_{i}(x)$, $\lambda_{a}=\lambda_{a}(x)$ is a solution, so is $y_{i}=e_{i}(x), \lambda_{\alpha}=c \lambda_{a}(x)$, where $c$ is any constant. Without loss of generality, it is assumed for the normal case that the $\lambda_{a}(x)$ are so chosen as to make $\left[\partial F / \partial y_{0}^{\prime}\right]^{x=x_{2}}$ equal to minus one.

\section{An Auxiliary Theorem on Differential Equations}

A certain problem in differential equations recurs several times in the course of this investigation. For clearness, as well as for brevity, it seems well to preface the study of the properties of the minimizing arc by a brief exposition of this problem and of the results obtained for it by applying the theorems on differential equations.

Consider an arc $A$

$$
y_{i}=y_{i}(x) \quad(\alpha \leqq x \leqq \beta)
$$

which is of class $D^{\prime}$, that is, one which is continuous and consists of a finite number of $\operatorname{arcs} A_{\tau}(\tau=1,2, \cdots, t)$ each of class $C^{\prime}$, and let the values of $x$ defining the end-points of $A_{\tau}$ be $x_{\tau-1}$ and $x_{\tau}$. Further suppose that each $A_{\tau}$ satisfies a system of differential equations

$$
\frac{d y_{i}}{d x}=f_{\tau i}\left(x, y_{0}, \cdots, y_{n}\right)
$$

for which the functions $f_{\tau_{0}}, \cdots, f_{\tau n}$ are of class $C^{\prime}$ in a neighborhood of the values $\left(x, y_{0}, \cdots, y_{n}\right)$ on $A_{\tau}$. Then through each point $\left(x, y_{0}, \cdots, y_{n}\right)$ $=\left(\xi, \eta_{0}, \cdots, \eta_{n}\right)$ of a sufficiently small neighborhood $(A)_{\delta}$ of $A$ there passes one and but one arc

$$
y_{i}=Y_{i}\left(x, \xi, \eta_{0}, \cdots, \eta_{n}\right)
$$

with the following properties:

1. When $\left(\xi, \eta_{0}, \cdots, \eta_{n}\right)$ is fixed in $(A)_{\delta}$ the $n+1$ functions $Y_{i}$ are of class $C^{\prime}$ in $x$ on each interval $x_{\tau-1} x_{\tau}$ and continuous in the whole interval $\alpha \leqq x \leqq \beta$.

2. On each interval $x_{\tau-1} x_{\tau}$ the functions $Y_{i}$ satisfy the corresponding differential equations (5).

3. For any set of values $\left(x, \xi, \eta_{0}, \cdots, \eta_{n}\right)$ such that $x_{\tau-1} \leqq x \leqq x_{\tau}$, and $\left(\xi, \eta_{0}, \cdots, \eta_{n}\right)$ is in $(A)_{\delta}$, the functions $Y_{i}$ and $Y_{i}^{\prime}=\partial Y_{i} / \partial x$ are of class $C^{\prime}$ in $x, \xi, \eta_{0}, \cdots, \eta_{n}$.

This theorem is true by known theorems on differential equations if $A$ consists of a single arc of class $C^{\prime} .^{*}$ For the more general case the constant $\delta$

* See Bolza, Vorlesungen über Variationsrechnung, p. 168. Bliss, The solutions of differential equations of the first order as functions of their initial values, A $\mathrm{n}$ a l s of $\mathrm{M}$ a t h e m a t i c s, 2d Series, vol. 6 (1905), p. 58. 
may be taken so small that if $\left(\xi, \eta_{0}, \cdots, \eta_{n}\right)$ is in $(A)_{\delta}$ and $\xi$ on the interval $x_{\tau-1} x_{\tau}$ then there passes through $\left(\xi, \eta_{0}, \cdots, \eta_{n}\right)$ one and but one solution (6) of the equations (5) with the properties of the theorem on $x_{\tau-1} x_{\tau}$. If $\delta$ is again restricted suitably, the initial values

$\left(x, y_{0}, \cdots, y_{n}\right)=\left[x_{\tau}, Y_{0}\left(x_{\tau}, \xi, \eta_{0}, \cdots, \eta_{n}\right), \cdots, Y_{n}\left(x_{\tau}, \xi, \eta_{0}, \cdots, \eta_{n}\right)\right]$

determine uniquely an arc satisfying the differential equations

$$
\frac{d y_{i}}{d x}=f_{\tau+1}, i(x, y)
$$

on the interval $x_{\tau} x_{\tau+1}$, and these will form with those for $x_{\tau-1} x_{\tau}$ an arc having the properties of the theorem on $x_{\tau-1} x_{\tau+1}$. By successive extensions of this sort an arc (6) arises which has the properties of the theorem on the whole interval $\alpha \leqq x \leqq \beta$.

\section{The Euler-Lagrange Equations and the Corner-point Condition}

Hahn has proved for the Mayer problem the theorem that a minimizing curve assumed to be of class $C^{\prime}$ is at least of class $C^{\prime \prime}$ in the neighborhood of a point for which the determinant $R\left(x, e, e^{\prime}, \lambda\right)$ of $\S 1$ is different from zero.* The foundation of his proof lies in an integration by parts analogous to that employed by du Bois-Reymond. In this section these methods will be extended to the case in which the minimizing arc is assumed to be of class $D^{\prime}$. The result of the study is to establish the first necessary condition for a minimum in what may be called the "du Bois-Reymond" form

$$
\int_{x_{1}}^{x} G_{i} d x-F_{i}=k_{i}
$$

where the $k$ 's are constants. The Euler-Lagrange equations and the continuity conditions necessary at a corner of the minimizing arc are necessary corollaries. The following lemmas lead to the du Bois-Reymond equations.

Lемма 1. Consider a minimizing arc $E_{12}$ for the Mayer problem which is of class $D^{\prime}$. Then, however the functions $\eta_{r}(x)$ may be chosen of class $D^{\prime}$, the equations of variation determine uniquely a set $\eta_{a}(x)$ of class $D^{\prime}$ vanishing for $x=x_{1}$ and such that the complete set $\eta_{i}(x)$ are admissible variations for $E$.

The substitution of the given functions $\eta_{r}$ in the equations (2) of $\S 1$ gives a set of differential equations for the functions $\eta_{a}$. If the values of $x$ where $E$ and the functions $\eta_{r}$ have corners are denoted by $x_{\tau}$, then these equations when solved for the derivatives $\eta_{a}^{\prime}$ give a system of the type (5) and the existence-theorem described in $\$ 2$ justifies the conclusion of the lemma.

* Monatshefte für Mathematik und Physik, vol. 14 (1903), p. 325. Mathematische Annalen, vol. 63 (1907), p. 266. 
Lемма 2. With every matrix $\left\|\eta_{a r}\right\|$ having elements which are of class $D^{\prime}$ and which vanish at $x_{1}$ and $x_{2}$ there is associated a matrix $\left\|n_{a i}\right\|$ the rows of which are admissible variations vanishing at $x_{1}$. The rows of the matrix $\left\|\eta_{a \beta}\right\|$ are determined from those of $\left\|\eta_{a r}\right\|$ as described in Lemma 1.

Lемма 3. If $E$ is a minimizing arc, then in the matrix \| $\eta_{a i} \|$ of Lemma 2 the determinant $\left|\eta_{a \beta}\left(x_{2}\right)\right|$ always vanishes, however the matrix $\left\|\eta_{a r}\right\|$ may have been chosen.

Suppose that for some choice of the matrix $\left\|\eta_{a r}\right\|$ with elements of class $D^{\prime}$ vanishing at $x_{1}$ and $x_{2}$ the associated matrix $\left\|\eta_{a i}\right\|$ is such that the determinant $\left|\eta_{a \beta}\left(x_{2}\right)\right|$ is different from zero. Define a set of functions $Y_{r}$ by the equations

$$
Y_{r}\left(x, \epsilon_{0}, \epsilon_{1}, \cdots, \epsilon_{m}\right)=e_{r}(x)+\epsilon_{0} \eta_{0 r}(x)+\cdots+\epsilon_{m} \eta_{m r}(x),
$$

and consider the equations

$$
\phi_{a}\left(x, y_{0}, \cdots, y_{m}, Y_{m+1}, \cdots, Y_{n}, y_{0}^{\prime}, \cdots, y_{m}^{\prime}, Y_{m+1}^{\prime}, \cdots, Y_{n}^{\prime}\right)=0 .
$$

If the values of $x$ defining corners of the curve $E$ and of the functions $\eta_{a i}$ are denoted by $x_{\sigma}$, then, since the determinant $\left|\psi_{\alpha \beta}\right|$ is different from zero along $E$, these equations may be solved for the derivatives $y_{a}^{\prime}$, and the resulting equations together with the $m+1$ equations $d \epsilon_{a} / d x=0$ will be of the type (5) considered in $\S 2$, with the known solution $y_{a}=e_{a}(x), \epsilon_{a}=0$. There is therefore a constant $\delta$ such that the initial conditions $y_{a}\left(x_{1}\right)=y_{a 1}$, $\left|\epsilon_{a}\right|<\delta$ yield a set of solutions $Y_{a}\left(x, \epsilon_{0}, \cdots, \epsilon_{m}\right), \epsilon_{\alpha}=$ constant, which with the functions $(7)$ above form an $(m+1)$-parameter family

$$
y_{i}=Y_{i}\left(x, \epsilon_{0}, \cdots, \epsilon_{m}\right)
$$

with continuity properties similar to those of the solutions of equations (5) of $\S 2$, and containing the arc $E$ for the values $x_{1} \leqq x \leqq x_{2},\left(\epsilon_{0}, \cdots, \epsilon_{m}\right)$ $=(0, \cdots, 0)$. Furthermore, along the arc $E, \partial Y_{i} / \partial \epsilon_{\beta}=\eta_{\beta i}$. For by differentiating with respect to $\epsilon_{\beta}$ the identities $\phi_{\alpha}\left(x, Y, Y^{\prime}\right)=0$, it follows that for a fixed $\epsilon_{\beta}$ the functions $\partial Y_{i} / \partial \epsilon_{\beta}$ along $E$ are solutions of the equations of variation (2) for $E$. From (7) it appears that the last $n-m$ of these derivatives are the functions $\eta_{\beta r}$. The first $m+1$ of them have initial values zero at $x=x_{1}$, since all the arcs (8) pass through the point $\left(x_{1}, y_{01}\right.$, $\left.\cdots, y_{n 1}\right)$; hence these first $m+1$ derivatives are the solutions $\eta_{a \beta}$ of the equations of variation for $E$ uniquely determined by these conditions.

Now suppose that $\epsilon_{0}, \cdots, \epsilon_{m}$ are determined as functions of another parameter $\epsilon$ in such a way that at $x=x_{2}$

$$
Y_{0}\left(x_{2}, \epsilon_{0}, \cdots, \epsilon_{m}\right)=y_{02}+\epsilon ; \quad Y_{p}\left(x_{2}, \epsilon_{0}, \cdots, \epsilon_{m}\right)=y_{p 2} .
$$

This is possible; for $\left(\epsilon, \epsilon_{0}, \cdots, \epsilon_{m}\right)=(0,0, \cdots, 0)$ is a solution-point 
of the system near which the functions are of class $C^{\prime}$ and at which the determinant

$$
\left|\left[\partial Y_{\alpha} / \partial \epsilon_{\beta}\right]^{x=x_{2}}\right|=\left|\eta_{\beta a}\left(x_{2}\right)\right| \neq 0 .
$$

Thus there is constructed a one-parameter family of arcs satisfying the differential equations of the Mayer Problem, having the given initial and end-values, but such that $\left[\partial Y_{0}\left(x_{2}, \epsilon\right) / \partial \epsilon\right]$ at $\epsilon=0$ is equal to unity, which contradicts the hypothesis that $e_{0}(x)$ is a minimum. The conclusion of the lemma must, therefore, be true.

TheOREM. If $E_{12}$ is a minimizing arc of class $D^{\prime}$ for the problem of Mayer as here proposed, then there exists a set of functions $\lambda_{a}$ of class $C^{\prime}$ except for values of $x$ defining the corners of $E$ where the $\lambda$ 's may be discontinuous, and $a$ set of constants $k_{i}$ such that the equations

$$
G_{i}=\int_{x_{1}}^{x} F_{i} d x+k_{i}
$$

are satisfied at every point of $E_{12}$.

Suppose the matrix $\left\|\eta_{a r}\right\|$ has been chosen and the matrix $\left\|\eta_{a i}\right\|$ determined as in Lemma 2. Let the functions $\lambda_{a}(x)$ be subject only to the hypothesis that they are of class $C$ in the interior of every interval on which $E$ is of class $C^{\prime}$ and have well defined right and left limits at the corners of $E$. Introduce the notations

$$
F\left(x, y, y^{\prime}, \lambda\right)=\sum_{a} \lambda_{a} \phi_{a}, \quad F_{i}=\partial F / \partial y_{i}, \quad G_{i}=\partial F / \partial y_{i}^{\prime} .
$$

Multiply the equations of variation (2) by the respective $\lambda$ 's and add. The resulting equation may be written

$$
\sum_{i}\left(F_{i} \eta_{i}+G_{i} \eta_{i}^{\prime}\right)=0
$$

the arguments of the derivatives of $F$ being those of $E$. The left-hand member of this equation may be integrated between $x_{1}$ and $x_{2}$; and the terms $F_{i} \eta_{i}$ may be integrated by parts since at every point of $\left[x_{1} x_{2}\right]$ the function $\sum_{i} \eta_{i}(x) \int_{x_{1}}^{x} F_{i} d x$ has a finite forward and backward derivative. ${ }^{*}$ Since $\eta_{i}\left(x_{1}\right)=\eta_{r}\left(x_{2}\right)=0$, the result of this operation is

$$
\sum_{a} \eta_{a}\left(x_{2}\right) \int_{x_{1}}^{x_{2}} G_{a} d x+\sum_{i} \int_{x_{1}}^{x_{2}}\left(G_{i}-\int_{x_{1}}^{\infty x} F_{i} d x\right) \eta_{i}^{\prime} d x=0 .
$$

Now, introduce a new set of functions $v_{a}(x)$ by means of the equations

$$
v_{a}=\sum_{\beta} \lambda_{\beta} \psi_{\beta a}=G_{a}
$$

where the arguments of the derivatives $\psi_{\beta a}$ are those of $E$. Since the deter-

* Cf. de la Vallće Poussin, Cours d'analyse infinitesimal, § 282, p. 263. 
minant $\left|\psi_{a \beta}\right|$ is different from zero along $E$, these equations may be solved for the functions $\lambda_{a}$ in terms of the functions $v_{\alpha}$. The functions $v_{\alpha}$ are to be so determined that they will satisfy the linear differential equations

$$
\frac{d v_{a}}{d x}=F_{\alpha}=\sum_{\beta} \lambda_{\beta} \phi_{\beta a}=\sum_{\gamma} a_{a \gamma} v_{\gamma},
$$

whose last members are obtained from the second by substituting the values of $\lambda_{a}$ in terms of $v_{a}$. These equations belong to the type (5) considered in $\S 2$.

The determinant $\left|\eta_{\alpha \beta}\left(x_{2}\right)\right|$ is zero, however the rest of the matrix is chosen. Suppose the matrix has been chosen so that this determinant has the maximum rank possible and denote the rank by $q$. Then it is possible to determine constants $p_{a}$ not all zero such that the equation

$$
\sum_{\beta} p_{\beta} \eta_{\beta}\left(x_{2}\right)=0
$$

holds for every row of the determinant $\left|\eta_{\beta a}\right|$. Since $q$ is the maximum rank attainable, the same relation is true for any admissible set of variations $\eta_{i}(x)$ for which $\eta_{i}\left(x_{1}\right)=\eta_{r}\left(x_{2}\right)=0$. The $p_{a}$ are unique (save for an arbitrary factor), if the determinant $\left|\eta_{\beta a}\left(x_{2}\right)\right|$ is of rank $m$.

As initial conditions for the solutions of the equations (12) take $v_{a}\left(x_{2}\right)=p_{a}$. A unique continuous solution is thus determined as in $\S 2$ and the functions $\lambda_{a}$ are determined in terms of the functions $v_{a}$ by means of equations (11). It is not possible that for any value of $x$ on the interval $\left[x_{1} x_{2}\right]$ all the functions $\lambda_{a}$ should vanish simultaneously, for by equations (11) this would involve a simultaneous vanishing of the functions $v_{a}$. The differential equations determining $v_{a}$ are linear, and the constants $p_{a}$ not all zero, so this is not possible. It is also to be noted that, while the functions $v_{a}$ are of class $D^{\prime}$ with corners at the values of $x$ defining corners of $E$, the functions $\lambda_{a}$ may have finite discontinuities precisely for those values of $x$ for which $E$ has corners, but are of class $C^{\prime}$ between those values.

From equations (11) and (12) it follows that the functions $\lambda_{a}$ satisfy the equations

$$
G_{a}=\int_{x_{1}}^{x} F_{a} d x+k_{a}
$$

where the $k_{a}$ are constants, since the functions $v_{a}$ are continuous. When the functions $\lambda_{a}$ thus obtained are substituted in equations (10) it is found that

$$
\sum_{a} \eta_{a}\left(x_{2}\right)\left(\int_{x_{1}}^{x_{2}} F_{a} d x+k_{a}\right)+\sum_{r} \int_{x_{1}}^{x_{2}} \eta_{r}^{\prime}\left(G_{r}-\int_{x_{1}}^{x} F_{r} d x\right) d x=0 .
$$

But the first sum vanishes, since the expressions in parentheses are the values $v_{a}\left(x_{2}\right)=p_{a}$, and the second sum must also be zero for every choice of the 
functions $\eta_{r}(x)$ of class $C^{\prime}$ and vanishing at $x_{1}$ and $x_{2}$. The functions in parentheses in the second sum have at most a finite number of finite discontinuities. A set $\left\|\eta_{r}\right\|$ may be chosen such that every $\eta_{r}$ except one is identically zero, and Whittemore's theorem is immediately applicable. ${ }^{*}$ It follows that the expression in parentheses must be equal to constants $k_{r}$, which completes the proof of the theorem.

COROLlaRY 1. If the arc $E_{12}$ is normal for the minimizing of $y_{0}(x)$, then the functions $\lambda_{a}$ of the preceding theorem are unique except for a constant factor.

On a normal arc the rank $m$ is always obtainable for the determinant $\left|\eta_{a \beta}\left(x_{2}\right)\right|$ of Lemma 3 ; for otherwise, there would be no choice of sets of admissible variations for which the matrix (4) of $\S 1$ is of rank $2 n+1$. As noted in the proof of the theorem, this makes the constants $p_{a}$ unique save for an arbitrary factor. Since these constants are initial conditions for the functions $v_{a}$, the uniqueness of these functions and consequently of the functions $\lambda_{a}$ is immediately demonstrated.

Corollary 2. At every point of $E$ which is not a corner the functions $G_{i}$ are of class $C^{\prime}$ and the Euler-Lagrange equations

$$
F_{i}-\frac{d}{d x} G_{i}=0 ; \quad \phi_{a}=0
$$

are satisfied. If at such a point the determinant $R$ of $\S 1$ is different from zero, then $E$ is also of class $C^{\prime \prime}$ near the point in question, and the terms $d G_{i} / d x$ of these equations can be differentiated out.

To demonstrate the second statement, the equations (9) of the theorem together with the equations $\phi_{a}=0$ may be regarded as a set of $n+m+2$ equations in the variables $x, y_{i}^{\prime}, \lambda_{a}$, where the functions $y_{i}=e_{i}(x)$, $y_{i}^{\prime}=e_{i}^{\prime}(x), \lambda_{a}=\lambda_{a}(x)$ belonging to the arc $E$ are substituted in $F_{i}$, and only the substitutions $y_{i}=e_{i}(x)$ are made in the functions $G_{i}$ and $\phi_{a}$. The equations in $x, y_{i}^{\prime}, \lambda_{a}$ so formed have the solution $y_{i}^{\prime}=e_{i}^{\prime}(x), \lambda_{a}=\lambda_{a}(x)$, and their functional determinant for the variables $y_{i}^{\prime}, \lambda_{a}$ is the determinant $R$. Near a point where $E$ is of class $C^{\prime}$ the functions of $x, y_{i}^{\prime}$, and $\lambda_{a}$ in these equations are of class $C^{\prime}$ and, if further, $R$ is different from zero, the usual theorems of implicit function theory show that the solution $y_{i}^{\prime}=e_{i}^{\prime}(x)$, $\lambda_{a}=\lambda_{a}(x)$ are of class $C^{\prime}$, so that $E$ is of class $C^{\prime \prime}$.

Corollary 3. The Corner-Point Condition. At every corner-point $x=x_{0}$ of a minimizing arc $E$ of class $D^{\prime}$ the condition

$$
G_{i}\left(x_{0}-0\right)=G_{i}\left(x_{0}+0\right)
$$

must be satisfied, the arguments of the derivatives $G_{i}$ being those of $E$.

Corollary 4. If an arc $E_{12}$ of class $C^{\prime}$ is a normal solution of the Euler-

\footnotetext{
*Annals of Mathematics, 2d Series, vol. 2 (1900-1901), p. 133.
} 
Lagrange equations (13), then the derivative $G_{0}$ is different from zero at $x=x_{2}$ on $E$, and the multipliers $\lambda_{a}(x)$ may be altered by a common constant factor so as to make $G_{0}\left(x_{2}\right)$ equal to any desired constant.

Every admissible variation of $E$ satisfies the equation

$$
\sum_{i}\left(F_{i} \eta_{i}+G_{i} \eta_{i}^{\prime}\right)=\sum_{i}\left[\left(F_{i}-\frac{d}{d x} G_{i}\right) n_{i}+\frac{d}{d x} G_{i} \eta_{i}\right]=0 .
$$

Since the arc $E$ satisfies the Euler-Lagrange equations, it follows that

$$
\sum_{i}\left[G_{i}\left(x_{2}\right) \eta_{i}\left(x_{2}\right)-G_{i}\left(x_{1}\right) \eta_{i}\left(x_{1}\right)\right]=0 .
$$

Since the functions $\lambda_{a}$ do not vanish simultaneously and the determinant $\left|\psi_{a \beta}\right|$ is everywhere different from zero, it follows that the derivatives $G_{i}$ do not vanish simultaneously on $E$. If $G_{0}\left(x_{2}\right)$ were zero, the equations (15) would hold in particular for the variations in each column of the determinant different from zero in the matrix (4). Since this determinant is different from zero, this is not possible.

A set of multipliers $\lambda_{a}$ can be altered by the same constant factor without destroying their usefulness, and since $G_{0}$ is linear in the $\lambda$ 's, these constants can be so chosen as to give $G_{0}\left(x_{2}\right)$ any value desired. It is convenient so to choose the multipliers $\lambda_{a}$ that $G_{0}\left(x_{2}\right)=-1$ and from this point on, it will be understood that such a choice of multiplier functions has been made.

\section{The Necessary Conditions of Weierstrass and Legendre}

In deducing a necessary condition analogous to that of Weierstrass the minimizing arc is supposed to be of class $D^{\prime}$ and to satisfy the Euler-Lagrange equations (13) and the corner-point condition (14) of $\S 3$. Furthermore every arc $\left[x_{3} x_{2}\right]$ of $E$ is supposed to be a normal arc.

The method here employed to establish the condition involves the use of a one-parameter family of curves with certain special properties. The extremal $E$ is cut at a point $P_{3}$ by a curve $I$. Then it is required to show that through the points $P_{4}$ lying on $H$ in the neighborhood of $P_{3}$ there passes a one-parameter family of curves $V$ of the following nature. 1. Each curve $V$ is a solution of the differential equations $\phi_{a}=0$. 2. The projection in the space $\left(x, y_{1}\right.$, $\left.\cdots, y_{n}\right)$ of that point of $V$ which has the $x$-coördinate $x_{2}$ coincides with the projection of the corresponding point of $E$; 3 . When $P_{4}$ falls on $P_{3}, V$ coincides with $E$. The situation in three-space is illustrated in the figure. The arc $P_{1} P_{2} P_{3}$ represents the arc $E$ with projection $P_{1}^{\prime} P_{2}^{\prime} P_{3}^{\prime}$ in the $X Y_{1^{-}}$ plane. $\quad P_{3} P_{4}$ is the arc $H$ and $P_{4} P_{2}^{\prime \prime}$ an arc $V$ of the family required.

To construct such a family let $y_{i}=h_{i}(x)$ be an arc of class $C^{\prime}$ for values of $x$ near $x_{3}$ and such that $h_{i}\left(x_{3}\right)=e_{i}\left(x_{3}\right)$. Since $E$ is normal on $x_{3} x_{2}$ there 
exist $2 n+1$ sets of admissible variations $\eta_{\sigma i}(\sigma=1,2, \cdots, 2 n+1)$ of class $C^{\prime}$ such that

$$
\left|\eta_{\sigma i}\left(x_{3}\right) \eta_{\sigma p}\left(x_{2}\right)\right| \neq 0 \text {. }
$$

In the rows of the matrix $i$ and $p$ are variable, while $\sigma$ in the columns has the

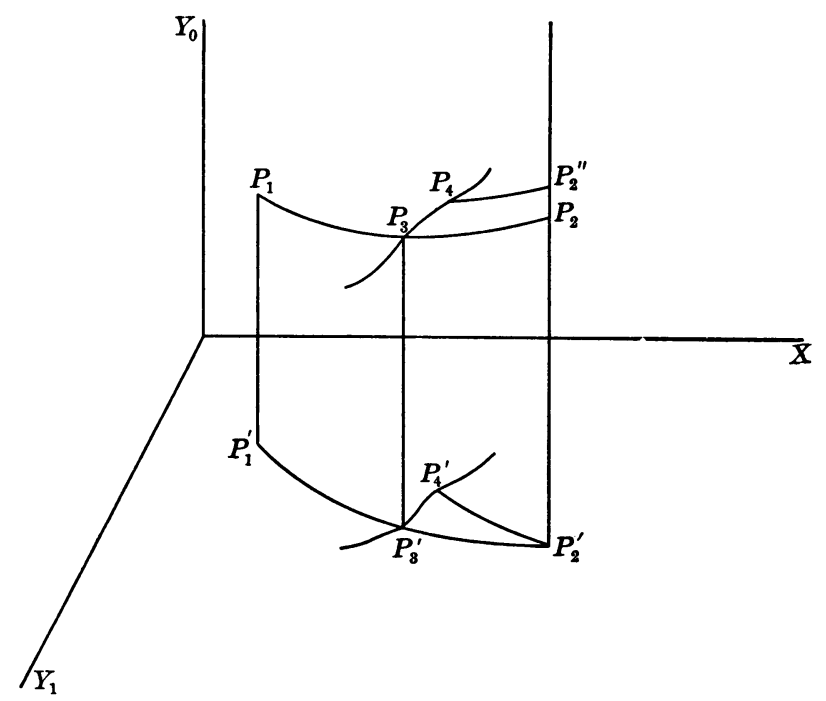

range indicated in $\S 1$. Then define $n-m$ functions $Y_{r}\left(x, \epsilon_{1}, \cdots, \epsilon_{2 n+1}\right)$ as follows

$$
Y_{r}(x, \epsilon)=e_{r}(x)+\sum_{\sigma} \epsilon_{\sigma} \eta_{\sigma r}(x) .
$$

Substitute from (17) for $y_{r}$ and $y_{r}^{\prime}$ in the equations $\phi_{a}=0$ and use the initial conditions

$$
Y_{a}\left(x_{3}, \epsilon_{1}, \cdots, \epsilon_{2 n+1}\right)=h_{a}\left(x_{3}\right)+\sum_{\sigma} \epsilon_{\sigma} \eta_{\sigma a}\left(x_{3}\right) .
$$

The solutions $Y_{a}\left(x, \epsilon_{1}, \cdots, \epsilon_{2 n+1}\right)$ obtained for $y_{a}$ by the well-known existence theorems for differential equations form with the functions (17) a $(2 n+1)$ parameter family

$$
y_{i}=Y_{i}\left(x, \epsilon_{1}, \cdots, \epsilon_{2 n+1}\right) .
$$

This family contains the arc $E$ for $x_{1} \leqq x \leqq x_{2}, \epsilon_{1}=\cdots=\epsilon_{2 n+1}=0$, and in a neighborhood of these values the functions $Y_{i}$ and $Y_{i}^{\prime}$ are of class $C^{\prime}$ in all variables. Furthermore, by reasoning similar to that of Lemma 2 of $\S 3$, it appears that along $E$ the values of the derivatives $\partial Y_{i} / \partial \epsilon_{\sigma}$ are precisely the functions $\eta_{\sigma i}(x)$, since this is from (16) clearly true when $i$ has a value on the range of $r$ and since, when $i$ has a value on the range of $\alpha$, the initial conditions (18) show that $\partial Y_{\sigma} / \partial \epsilon_{\sigma}$ has on $E$ for $x=x_{3}$ the value $\eta_{\sigma a}\left(x_{3}\right)$.

The parameters $\epsilon_{\sigma}$ are now to be determined as functions of $x_{4}$ in such a way that 


$$
Y_{i}\left(x_{4}, \epsilon_{1}, \cdots, \epsilon_{2 n+1}\right)=h_{i}\left(x_{4}\right), \quad Y_{p}\left(x_{2}, \epsilon_{1}, \cdots, \epsilon_{2 n+1}\right)=e_{p}\left(x_{2}\right) .
$$

These equations have the solution $x_{4}=x_{3}, \epsilon_{1}=\cdots=\epsilon_{2 n+1}=0$, near which the functions which they involve are of class $C^{\prime}$ and at which their functional determinant is the determinant (16), which is different from zero. The parameters $\epsilon_{\sigma}$ may therefore be determined as functions of $x_{4}$ of class $C^{\prime}$ which for $x_{4}=x_{3}$ take the values $\epsilon_{1}=\cdots=\epsilon_{2 n+1}=0$, and which satisfy equations (19). If these solutions are denoted by $\epsilon_{\sigma}\left(x_{4}\right)$ then the arc

$$
y_{i}=v_{i}\left(x, x_{4}\right)=Y_{i}\left(x, \epsilon_{1}\left(x_{4}\right), \cdots, \epsilon_{2 n+1}\left(x_{4}\right)\right)
$$

is the arc $V$ which was sought. For

$$
\begin{aligned}
v_{i}\left(x, x_{3}\right) & =Y_{i}(x, 0, \cdots, 0)=e_{i}(x), \\
v_{i}\left(x_{4}, x_{4}\right) & =Y_{i}\left[x_{4}, \epsilon_{1}\left(x_{4}\right), \cdots, \epsilon_{2 n+1}\left(x_{4}\right)\right]=h_{i}\left(x_{4}\right), \\
v_{p}\left(x_{2}, x_{4}\right) & =Y_{p}\left[x_{2}, \epsilon_{1}\left(x_{4}\right), \cdots, \epsilon_{2 n+1}\left(x_{4}\right)\right]=e_{p}\left(x_{2}\right) ;
\end{aligned}
$$

and $V$ satisfies also the differential equations $\phi_{a}=0$.

The theorem on the Weierstrass necessary condition may be stated as follows:

Theorem. Let $E$ be a minimizing arc of class $C^{\prime}$ which is normal on every interval $x_{3} \leqq x \leqq x_{2}$. Let $\left(y_{0}^{\prime}, \cdots, y_{n}^{\prime}\right)$ be a direction such that at the set of values $x_{3}, e_{0}\left(x_{3}\right), \cdots, e_{n}\left(x_{3}\right), y_{0}^{\prime}, \cdots, y_{n}^{\prime}$, the equations $\phi_{a}=0$ are satisfied and the matrix $\left\|\partial \phi_{a} / \partial y_{i}^{\prime}\right\|$ of rank $m+1$. Then, if $E$ makes the value $e_{0}\left(x_{2}\right)$ a strong relative minimum, it is necessary that

$E\left[x_{3}, e_{0}\left(x_{3}\right), \cdots, e_{n}(x), e_{0}^{\prime}\left(x_{3}\right), \cdots, e_{n}^{\prime}\left(x_{3}\right), y_{0}^{\prime}, \cdots, y_{n}^{\prime}, \lambda_{0}\left(x_{3}\right)\right.$,

where the function $E$ is defined to be

$$
\left.\cdots, \lambda_{m}\left(x_{3}\right)\right] \geqq 0 \text {, }
$$

$$
\begin{aligned}
E=F\left(x, e_{0}, \cdots,\right. & \left.e_{n}, y_{0}^{\prime}, \cdots, y_{n}^{\prime}, \lambda_{0}, \cdots, \lambda_{m}\right) \\
& -F\left(x, e_{0}, \cdots, e_{n}, e_{0}^{\prime}, \cdots, e_{n}^{\prime}, \lambda_{0}, \cdots, \lambda_{m}\right) \\
& -\sum_{i}\left(y_{i}^{\prime}-e_{i}^{\prime}\right) G_{i}\left(x, e_{0}, \cdots, e_{n}, e_{0}^{\prime}, \cdots, e_{n}^{\prime}, \lambda_{0}, \cdots, \lambda_{m}\right) .
\end{aligned}
$$

Through the point $P_{3}=\left[x_{3}, e\left(x_{3}\right)\right]$ construct a curve $H$ with equations $y_{i}=h_{i}(x)$ which is of class $C^{\prime}$ and satisfies the conditions

$$
\phi_{a}\left(x, h_{0}, \cdots, h_{r}, h_{0}^{\prime}, \cdots, h_{n}^{\prime}\right)=0 ; \quad h_{i}\left(x_{3}\right)=e_{i}\left(x_{3}\right) ; \quad h_{i}^{\prime}\left(x_{3}\right)=y_{i}^{\prime} .
$$

This is always possible, since under the hypotheses for $y_{i}^{\prime}$ the equations $\phi_{a}=0$ are solvable for $m+1$ of the variables $y_{i}^{\prime}$ near the values $x_{3}, e_{0}\left(x_{3}\right), \cdots$, $e_{n}\left(x_{3}\right), y_{0}^{\prime}, \cdots, y_{n}^{\prime}$ of the theorem. If we denote the corresponding $m+1$ functions among the $y$ 's by $u_{0}, u_{1}, \cdots, u_{m}$, then the differential equations so formed determine the $u$ 's uniquely when their initial values at $x=x_{3}$ are 
prescribed and the remaining $n-m$ functions $v_{1}, \cdots, v_{n-m}$ among the $y$ 's are chosen arbitrarily of class $C^{\prime}$ near $x=x_{3}$. If the initial values of the $v$ 's and their derivatives are the corresponding values among the values $e_{i}\left(x_{3}\right)$ and $y_{i}$, and, if the initial values of the $u$ 's are the values corresponding to them in the set $e_{i}\left(x_{3}\right)$, then the curve $y_{i}=h_{i}(x)$ determined by the functions $u_{0}, u_{1}, \cdots, u_{m}, v_{1}, \cdots, v_{n-m}$ will have the desired properties.

Let $P_{4}$ with $x$-coördinate $x_{4}$ lie on $H$ near $P_{3}$, and construct a family with equations (20) and properties (21) as described in the first paragraphs of this section. Then on the interval $x_{4} x_{2}$ it is true that the equations

$$
F\left(x, v_{0}, \cdots, v_{n}, v_{n}^{\prime}, \cdots, v_{n}^{\prime}, \lambda_{0}, \cdots, \lambda_{m}\right) \equiv 0,
$$

formed with the multipliers $\lambda$ belonging to $E$, are satisfied, since every arc $V$ satisfies the equations $\phi_{a}=0$. If this last equation is differentiated with respect to the parameter $x_{4}$ and $x_{4}$ set equal to $x_{3}$, it follows that

$$
\sum_{i}\left[F_{i} \eta_{i}+G_{i} \eta_{i}^{\prime}\right]=\sum_{i}\left[\left(F_{i}-\frac{d}{d x} G_{i}\right) \eta_{i}+\frac{d}{d x} G_{i} \eta_{i}\right]=0,
$$

where $\eta_{i}(x)$ denotes the function $\partial v_{i} / \partial x_{4}$ for $x_{4}=x_{3}$. The derivatives $F_{i}, G_{i}$ are those of $E$, since $V$ reduces to $E$ for $x_{4}=x_{3}$. Since $E$ is an extremal, it follows that $\sum_{i} G_{i} \eta_{i}$ is a constant and has the same value at $x=x_{2}$ and at $x=x_{3}$. By differentiating the second and third of equations (21) with respect to $x_{4}$ and setting $x_{4}=x_{3}$, it appears with the help of the first of equations (21) that

$$
\eta_{i}\left(x_{3}\right)+v_{i}^{\prime}\left(x_{3}, x_{3}\right)=\eta_{i}\left(x_{3}\right)+e_{i}^{\prime}\left(x_{3}\right)=h_{i}^{\prime}\left(x_{3}\right) ; \quad \eta_{p}\left(x_{2}\right)=0 .
$$

Hence, by equating the values of $\sum_{i} G_{i} \eta_{i}$ at $x_{2}$ and at $x_{3}$

$$
\eta_{0}\left(x_{2}\right)=-\left[\sum_{i}\left(h_{i}^{\prime}-e_{i}^{\prime}\right) G_{i}\right]^{x=x_{8}},
$$

since $G_{0}\left(x_{2}\right)=-1$. But, if $e_{0}(x)$ is a minimum, we must have

$$
\eta_{0}\left(x_{2}\right)=\left[\left(\partial v_{0} / \partial x_{4}\right]^{x_{4}=x_{8}} \geqq 0,\right.
$$

from which the conclusion of the theorem follows, since the second member of (23) differs from the $E$-function only by the terms

$$
\begin{aligned}
F\left(x, e_{0}, \cdots, e_{n}, y_{0}^{\prime}, \cdots, y_{n}^{\prime},\right. & \left.\lambda_{0}, \cdots, \lambda_{m}\right) \\
& -F\left(x, e_{0}, \cdots, e_{n}, e_{0}^{\prime}, \cdots, e_{n}^{\prime}, \lambda_{0}, \cdots, \lambda_{m}\right)
\end{aligned}
$$

which are both zero at $x=x_{3}$.

The Necessary Condition of Legendre. If $E$ is a minimizing arc satisfying the hypotheses of the last theorem, then at each point of $E$ the quadratic form

$$
\sum_{i j} R_{i j} \zeta_{i} \zeta_{j}
$$


is positive or zero for every set of values $\zeta_{0}, \cdots, \zeta_{n}$ satisfying the equations

$$
\sum_{i} \psi_{a i} \zeta_{i}=0
$$

Suppose $\zeta_{0}, \cdots, \zeta_{n}$ is such a set for a point of $E$ defined by the coördinate $x$. Define a set of functions

$$
Y_{r}^{\prime}(\epsilon)=e_{r}^{\prime}(x)+\epsilon \zeta_{r}(x) .
$$

Under the hypothesis that the determinunt $\left|\psi_{a \beta}\right|$ is different from zero the equations $\phi_{a}\left(x, e_{0}, \cdots, e_{n}, y_{0}^{\prime}, \cdots, y_{m}^{\prime}, Y_{m+1}^{\prime}, \cdots, Y^{\prime}\right)=0$ can be solved for $y_{a}^{\prime}=Y_{a}^{\prime}(\epsilon)$ in the neighborhood of the solution

$$
\epsilon=0, \quad Y_{a}^{\prime}=e_{a}^{\prime}(x) .
$$

Substitute the $Y_{i}^{\prime}(\epsilon)$ thus obtained for $y_{i}^{\prime}$ in the equations $\phi_{a}=0$, differentiate the result with respect to $\epsilon$, and place $\epsilon$ equal to zero. When the definition (24) is utilized, it appears that

$$
\sum_{\beta} \psi_{a \beta}\left[\partial Y_{\beta}^{\prime} / \partial \epsilon\right]^{\epsilon=0}+\sum_{r} \psi_{a r} \zeta_{r}=0 .
$$

From this equation with the hypothesis $\left|\psi_{\alpha \beta}\right| \neq 0$, it is arguable that for values of $i$ on the range $\alpha$, as well as for those on the range $r$,

$$
\left[\partial Y_{i}^{\prime} / \partial \epsilon\right]^{\epsilon=0}=\zeta_{i} .
$$

Since the functions $Y_{i}^{\prime}(\epsilon)$ satisfy the hypothesis for $y_{i}^{\prime}$ in the theorem on the Weierstrass condition, then

$$
\begin{aligned}
E(\epsilon) & =E\left(x, e_{0}, \cdots, e_{n}, e_{0}^{\prime}, \cdots, e_{n}^{\prime}, Y_{0}^{\prime}, \cdots, Y_{n}^{\prime}, \lambda_{0}, \cdots, \lambda_{m}\right) \\
& =F\left(x, e, Y^{\prime}, \lambda\right)-F\left(x, e, e^{\prime}, \lambda\right)-\sum_{i}\left(Y_{i}^{\prime}-e_{i}^{\prime}\right) G_{i}\left(x, e, e^{\prime}, \lambda\right) \geqq 0 .
\end{aligned}
$$

If $E(\epsilon)$ is expanded by Taylor's Theorem in the neighborhood of $\epsilon=0$, the coefficients $E(0), E^{\prime}(0)$ are seen to be equal to zero. Then

$$
E(\epsilon)=\epsilon^{2} \int_{0}^{1}(1-u) E^{\prime \prime}(u, \epsilon) d u \geqq 0 .
$$

As $\epsilon$ tends to zero, the function $E^{\prime \prime}(u, \epsilon)$ tends to the limit $\sum_{i j} R_{i j} \zeta_{i} \zeta_{j}$; and from continuity considerations the truth of the theorem is evident.

\section{The Second Variation and the Jacobi Condition}

In the remaining paragraphs of this paper a change of notation is made in the interest of simplicity of statement. Multipartite numbers and matrices will henceforth be denoted in all but a few exceptional cases by single symbols, the $(n+1)$-partite functions $y_{0}, \cdots, y_{n}, e_{0}, \cdots, e_{n}, \eta_{0}, \cdots, \eta_{n}$ by the 
respective symbols $y, e, \eta$; the $(m+1)$-partite functions $\phi_{0}, \cdots, \phi_{m}$, $\lambda_{0}, \cdots, \lambda_{m}$ by $\phi, \lambda$. Partial derivatives will be indicated by subscripts, so that the sets of functions $F_{0}, \cdots, F_{n}, G_{0}, \cdots, G_{n}$ are now denoted by $F_{y}, F_{y}^{\prime}$ respectively. The double subscript indicates a matrix, as

$$
F_{y^{\prime} y^{\prime}}=\left\|\partial^{2} F / \partial y_{i}^{\prime} \partial y_{j}^{\prime}\right\|=\left\|R_{i j}\right\| \text {. }
$$

The usual rules of combination and properties of products are assumed.*

From this point, it will be assumed that the minimizing arc $E$ is of class $C^{\prime}$ and that all along $E$ the determinant $R\left(x, e, e^{\prime}, \lambda\right)$ of $\S 1$ is different from zero. It is further supposed, as in $\S 4$, that $E$ is normal on every sub-interval $x_{3} x_{2}$ of the interval $x_{1} x_{2}$. From Corollary 3 of the theorem of $\S 3$, it appears that $E$ is thus of class $C^{\prime \prime}$.

Lemma 1. If functions $\eta(x)$ are a set of admissible variations of class $D^{\prime}$ such that $\eta_{i}\left(x_{1}\right)=\eta_{p}\left(x_{2}\right)=0$, then there exists a one-parameter family $Y(x, \epsilon)$ containing $E$ for $\epsilon=0$ and having the functions $\eta(x)$ as its set of variations along $E$.

A $(2 n+2)$-parameter family is first determined by methods analogous to those employed in the first paragraphs of $\S 4$. There are defined $n-m$ arbitrary functions

$$
Y_{r}\left(x, \epsilon, \epsilon_{1}, \cdots, \epsilon_{2 n+1}\right)=e_{r}(x)+\epsilon \eta_{r}(x)+\sum_{\sigma} \epsilon_{\sigma} \eta_{\sigma r}(x),
$$

the functions $\eta_{\sigma r}$ being the respective variations appearing in the non-vanishing determinant of the matrix (4). Substitutions from (25) for $y_{r}$ and $y_{r}^{\prime}$ in $\phi_{a}=0$ with the use of the initial conditions

$$
Y_{a}\left(x_{1}, \epsilon, \epsilon_{1}, \cdots, \epsilon_{2 n+1}\right)=e_{\alpha}\left(x_{1}\right)+\sum_{a} \epsilon_{\sigma} \eta_{\sigma a}\left(x_{1}\right)
$$

completely determines the $Y_{a}\left(x, \epsilon, \epsilon_{1}, \cdots, \epsilon_{2 n+1}\right)$. The $\eta_{\sigma a}$ are, of course, again the functions appearing in the determinant from (4). The family $Y_{i}$ thus determined has variations $\eta_{i}$ for $\epsilon, \eta_{\sigma i}$ for $\epsilon_{\sigma}$ along $E$. Since the determinant from (4) is different from zero, it is possible to determine the $\epsilon_{\sigma}$ as functions of $\epsilon$ satisfying the equations

$$
Y_{i}\left(x_{1}, \epsilon, \epsilon_{1}, \cdots, \epsilon_{2 n+1}\right)=e_{i}\left(x_{1}\right) ; \quad Y_{p}\left(x_{2}, \epsilon, \epsilon_{1}, \cdots, \epsilon_{2 n+1}\right)=e_{p}\left(x_{2}\right) .
$$

The family

$$
Y\left[x, \epsilon, \epsilon_{1}(\epsilon), \cdots, \epsilon_{2 n+1}(\epsilon)\right]=Y(x, \epsilon)
$$

thus determined is the one required.

Suppose that $\eta(x)$ is a set of admissible variations of class $D^{\prime}$ for $E$ with

* See Bliss, "The solutions of differential equations of the first order as functions of their initial values," A n nals of M a themati cs, 2d Series, vol. 6.

Trans. Am. Matb. Soc. $\%$ 
$\eta\left(x_{1}\right)=\eta_{p}\left(x_{2}\right)=0$ and that $Y(x, \epsilon)$ is an admissible family constructed as in the lemma. Define

$$
\zeta(x)=\left[\partial^{2} Y(x, \epsilon) / \partial \epsilon^{2}\right]^{\epsilon=0} .
$$

Since $Y(x, \epsilon)$ is an admissible family, $F\left(x, Y, Y^{\prime}\right)=0$ for every $\epsilon$. If this equation is differentiated twice with respect to $\epsilon$, and $\epsilon$ is put equal to zero, it follows that

$$
F_{y y} \eta \eta+2 F_{y y^{\prime}} \eta \eta^{\prime}+F_{y^{\prime} y^{\prime}} \eta^{\prime} \eta^{\prime}+F_{y} \zeta+F_{y^{\prime}} \zeta^{\prime}=0,
$$

the arguments of the partial derivatives of $F$ being, of course, those of $E$. It will be found convenient to denote the quadratic form $F_{y y} \eta \eta+2 F_{y y^{\prime}} \eta \eta^{\prime}$ $+F_{y^{\prime} y^{\prime}} \eta^{\prime} \eta^{\prime}$ by $\omega\left(x, \eta, \eta^{\prime}\right)$. From the use of equations (13) it appears that

$$
F_{y} \zeta+F_{y^{\prime}} \zeta^{\prime}=\frac{d}{d x} F_{y^{\prime}} \zeta
$$

Set $z=-F_{y^{\prime}} \zeta$. Equation (26) may then be written $-z^{\prime}+\omega(x, \eta, \eta)=0$. From the conditions on the family $Y(x, \epsilon)$ it follows that

$$
z\left(x_{1}\right)=0 ; \quad z\left(x_{2}\right)=-\left.F_{y_{0}^{\prime}} \zeta_{0}\right|^{x_{2}}=\zeta_{0}\left(x_{2}\right) .
$$

As has been noted in $\S 4, F_{y^{\prime}} \eta$ is a constant along $E$, so that the hypothesis $\eta_{p}\left(x_{2}\right)=0$ implies $\eta_{0}\left(x_{2}\right)=0$. If $e_{0}\left(x_{2}\right)$ is to be a minimum, it must be true that $z\left(x_{2}\right)=\zeta_{0}\left(x_{2}\right)$ is greater than or equal to zero.

The study of the second variation thus leads to an auxiliary Mayer problem, which will be referred to as the problem $\left(M^{\prime}\right)$ and which may be stated as follows:

$\left(M^{\prime}\right)$ Among all sets of functions $\eta(x), z(x)$ satisfying the equations

$$
\begin{aligned}
\chi\left(x, \eta, \eta^{\prime}\right) & =\phi_{y} \eta+\phi_{y}{ }^{\prime} \eta^{\prime}=0, \\
\psi\left(x, \eta, \eta^{\prime}, z^{\prime}\right) & =\omega\left(x, \eta, \eta^{\prime}\right)-z^{\prime}=0,
\end{aligned}
$$

and the conditions

$$
\eta\left(x_{1}\right)=z\left(x_{1}\right)=\eta\left(x_{2}\right)=0,
$$

to determine one making $z\left(x_{2}\right)$ a minimum.

The function $\chi$ is $(m+1)$-partite, so the problem involves $m+2$ equations in the $n+2$ variables $\eta=\eta_{0}, \eta_{1}, \cdots, \eta_{n}, z$. If $E$ is a minimizing arc for the original problem, it follows from the discussion above that zero is a minimum value for $z\left(x_{2}\right)$. Every minimizing arc, therefore, which gives to $z\left(x_{2}\right)$ the value zero must satisfy the necessary conditions for a minimum deduced in $\S \S 3$ and 4 .

Since the differential equations (27) are independent of $z$ and $z^{\prime}$ occurs only in the last equation and there linearly, the Lagrange multiplier-function 
associated with the function $\psi$ must be a constant. Call it $l$. Let the $(m+1)$-partite function $2 \mu$ represent the multipliers associated with the respective functions $\chi$. If $\Omega=l \psi+2 \mu \chi$, it must be true that at every point not a corner-point of the minimizing curve $z(x), \eta(x)$ the EulerLagrange equations

$$
\begin{aligned}
\Omega_{z}-\frac{d}{d x} \Omega_{z^{\prime}}=\frac{d}{d x} l & =0, & \Omega_{\eta}-\frac{d}{d x} \Omega_{\eta^{\prime}} & =0, \\
\Omega_{\mu}=2\left(\phi_{y} \eta+\phi_{y^{\prime}} \eta^{\prime}\right) & =0, & \Omega_{l}=\omega\left(x, \eta, \eta^{\prime}\right)-z^{\prime} & =0,
\end{aligned}
$$

are satisfied. The first equation shows that the multiplier $l$ of the function $\psi$ must be a constant, as was mentioned above, and this equation is satisfied in that case for every $\eta, z$. Any solution of equations (28) giving to $z\left(x_{2}\right)$ the value zero must at a corner satisfy the corner-point condition deduced in $\S 3$. This fact is made the basis of a proof of the Jacobi theorem for the original problem. The equations

$$
\Omega_{\eta}-\frac{d}{d x} \Omega_{\eta^{\prime}}=0, \quad \Omega_{\mu}=0
$$

may be called the Jacobi equations of the original Mayer problem. By a solution $\eta, \mu$ of equations (29) is always meant a set with $\eta$ of class $C^{\prime \prime}, \mu$ of class $C^{\prime}$ satisfying the equations (29) on the interval $x_{1} x_{2}$.

Lemma 2. If a solution $\eta, \mu$ of the Jacobi equations is such that for a value $x_{3}$ between $x_{1}$ and $x_{2} \eta\left(x_{3}\right)=\eta^{\prime}\left(x_{3}\right)=\mu\left(x_{3}\right)=0$, then the functions $\eta, \mu$ are identically zero.

If the second group of equations (29) is differentiated with respect to $x$, the result with the first group of (29) forms a set of equations solvable for the derivatives $\eta^{\prime \prime}, \mu^{\prime}$, since the determinant $R\left(x, e, e^{\prime}, \lambda\right)$ is different from zero. The system that results is of the form

$$
\frac{d \eta}{d x}=\eta^{\prime} ; \quad \frac{d \eta^{\prime}}{d x}=g\left(x, \eta, \eta^{\prime}, \mu\right) ; \quad \frac{d \mu}{d x}=h\left(x, \eta, \eta^{\prime}, \mu\right) .
$$

One solution of (30) is $\eta \equiv 0, \eta^{\prime} \equiv 0, \mu \equiv 0$. From the known theories of differential equations any solution assuming at an initial point $x=x_{3}$ values identical with those assumed by this solution must be identical with it. This proves the lemma.

THEOREM. If $E$ is a minimizing arc for the Mayer problem with the properties specified, then no solution $\eta=u(x), l=1, \mu=\rho(x)$ of the Jacobi equations can exist with $u\left(x_{1}\right)=u\left(x_{3}\right)=0\left(x_{1}<x_{3}<x_{2}\right)$ and the function $u$ not identically zero.

Suppose such a solution exists. Define $\eta, \mu$ as follows: When $x_{1} \leqq x \leqq x_{3}$, $\eta(x)=u(x)$, and $\mu(x)=\rho(x)$. When $x_{3} \leqq x \leqq x_{2}, \eta(x)=0$ and 
$\mu(x)=0$. The $\eta$ and $\mu$ thus defined are a solution of equations (29) giving $z\left(x_{2}\right)$ its minimum value zero. For

$$
\begin{aligned}
z\left(x_{2}\right)=\int_{x_{1}}^{x_{2}} \omega\left(x, \eta, \eta^{\prime}\right) d x=\int_{x_{1}}^{x_{3}} \omega\left(x, u, u^{\prime}\right) d x & \\
& =\frac{1}{2} \int_{x_{1}}^{x_{3}}\left(\omega_{u} u+\omega_{u^{\prime}} u^{\prime}\right) d x .
\end{aligned}
$$

The last step is possible, since $\omega\left(x, u, u^{\prime}\right)$ is a function homogeneous in $u, u^{\prime}$. Integrate by parts and obtain

$z\left(x_{2}\right)=\frac{1}{2}\left[\omega_{u^{\prime}} u\right]_{x_{1}}^{x_{3}}+\frac{1}{2} \int_{x_{1}}^{x_{3}}\left(\omega_{u}-\frac{d}{d x} \omega_{u^{\prime}}\right) u d x=\frac{1}{2} \int_{x_{1}}^{x_{3}}\left(\omega_{u}-\frac{d}{d x} \omega_{u^{\prime}}\right) u d x$.

Since $l=1, \eta=u(x), \mu=\rho(x)$ is a solution of the Jacobi equations (29), it follows that

$$
\omega_{u}-\frac{d}{d x} \omega_{u^{\prime}}=-2\left(\rho \phi_{y}-\frac{d}{d x} \rho \phi_{y^{\prime}}\right), \quad\left(\rho \phi_{y}-\frac{d}{d x} \rho \phi_{y^{\prime}}\right) u=-\frac{d}{d x}\left(\rho \phi_{y^{\prime}} u\right) .
$$

The expression for $z\left(x_{2}\right)$ may be written

$$
z\left(x_{2}\right)=\int_{x_{1}}^{x_{2}} \frac{d}{d x}\left(\rho \phi_{y^{\prime}}^{\prime} u\right) d x=\left[\rho \phi_{y^{\prime}} u\right]_{x_{1}}^{x_{3}} .
$$

By the conditions on $u$ this is zero.

But this solution $\eta, \mu$ has a corner-point at $x=x_{3}$. By the results of $\xi 3$ it is necessary that

$$
\left.\Omega_{\eta^{\prime}}\left(x, u, u^{\prime}, \rho\right)\right|^{x_{3}}=\Omega_{\eta^{\prime}}(x, 0,0,0) .
$$

Since $u(x), \rho(x)$ satisfy equations (29), the values assumed at $x_{3}$ must satisfy the system

$$
F_{y^{\prime} y^{\prime}} u^{\prime}\left(x_{3}\right)+\left.\rho\left(x_{3}\right) \phi_{y^{\prime}}\right|^{x_{3}}=0,\left.\quad\left(\phi_{y^{\prime}} u^{\prime}\right)\right|^{\mid x_{3}}=0 .
$$

By hypothesis the determinant $R\left(x, e, e^{\prime}, \lambda\right)$ is everywhere different from zero. So the equations (32) can be satisfied only if $u^{\prime}\left(x_{3}\right)=\rho\left(x_{3}\right)=0$. Since $u\left(x_{3}\right)=0$, it follows from the lemma that $u(x) \equiv 0, \rho(x) \equiv 0$. This establishes the theorem.

The conjugate point to $P_{1}$ on the arc $P_{1} P_{2}$ may be defined as follows: $P_{3}\left(x=x_{3}\right)$ is conjugate to $P_{1}\left(x=x_{1}\right)$, if there exist solutions $u(x), \rho(x)$ of equations (29) such that $u(x)$ is not identically zero and $u\left(x_{1}\right)=u\left(x_{3}\right)=0$.

The preceding discussion establishes the

JACOBI Theorem. If $E_{12}$ is a minimizing arc for the Mayer problem, no point conjugate to $P_{1}$ can lie between $P_{1}$ and $P_{2}$. 


\section{Theorems on the Solutions of the Jacobi Equations}

There remains the question of the actual determination of points conjugate to $P_{1}$. A study of the properties of the solutions of the Jacobi equations leads to the formation of a determinant whose zeros are the conjugate points required. It can then be shown that these points may also be determined as zeros of a determinant formed from the derivatives of solutions of the EulerLagrange equations. The series of theorems that follows will establish these facts.

Theorem 1. If $\eta=u(x), \mu=\rho(x)$ is a solution of the Jacobi equations (29) then $\eta=u(x), \mu=\rho(x)+k \lambda(x)$ is also a solution, where $\lambda$ is the multiplier-function appearing in the Euler equations for the Mayer problem and $k$ is any scalar constant.

The theorem is proved by substituting the proposed solution in the equations (29) and noting that $\lambda$ satisfies the Euler equations.

CoRollary. The equations (29) aamit the particular solution $\eta=0, \mu=\lambda$.

Theorem 2. If $\eta=u, \mu=\rho$ is a solution of the equations (29) and the arc $E_{12}$ is normal, then every solution $\mu$ associated with $\eta=u$ is of the form $\mu=\rho+k \lambda$, where $\lambda$ is the multiplier-function as before and $k$ is an arbitrary scalar constant.

Suppose equations (29) are satisfied by $\eta=u, \mu=\rho_{1}$ and also by $\eta=u$, $\mu=\rho_{2}$. Substitute in equations (29) and subtract the first result from the second. The equations obtained are

$$
\left(\rho_{1}-\rho_{2}\right) \phi_{y}-\frac{d}{d x}\left(\rho_{1}-\rho_{2}\right) \phi_{y^{\prime}}=0 .
$$

In $\S 3$ it was proved that for a normal arc the multipliers $\lambda$ are unique except for a constant factor. It follows that $\rho_{1}-\rho_{2}=k \lambda$.

Theorem 3. The Jacobi equations have $(2 n+1)$ solutions $u^{(\sigma)}$, $\rho^{(\sigma)}$ such that the identities

$$
\sum_{\sigma} c_{\sigma} u^{(\sigma)}(x) \equiv 0, \quad \sum_{\sigma} c_{\sigma} \rho^{(\sigma)}(x)+c \lambda(x) \equiv 0
$$

are satisfied only when all the constants $c_{\sigma}$, c are zero. In terms of $2 n+1$ linearly independent solutions of this sort every other solution is expressible in the form

$$
\eta(x)=\sum_{\sigma} c_{\sigma} u^{(\sigma)}(x), \quad \mu(x)=\sum_{\sigma} c_{\sigma} \rho^{(\sigma)}(x)+c \lambda(x) .
$$

It was noted in the proof of the lemma to $\$ 5$ that the equations (29) can be put in the form (30) and that a solution of these equations is uniquely determined when initial values of $x, \eta(x), \eta^{\prime}(x), \mu(x)$ are assigned. In particular, the initial values $x=x_{1}, \eta\left(x_{1}\right)=\eta^{\prime}\left(x_{1}\right)=\mu\left(x_{1}\right)=0$ are asso- 
ciated with the solution $\eta(x) \equiv 0, \mu(x) \equiv 0$. To determine $u^{(\sigma)}(x) \rho^{(\sigma)}(x)$ as in the theorem, initial values $u^{(\sigma)}\left(x_{1}\right), \rho^{(\sigma)}\left(x_{1}\right)$ are so selected that the determinant

$$
\left|\begin{array}{lc}
u_{i}^{(\sigma)}\left(x_{1}\right) & 0 \\
u_{r}^{(\sigma)}\left(x_{1}\right) & 0 \\
\rho_{a}^{(\sigma)}\left(x_{1}\right) & \lambda_{a}\left(x_{1}\right)
\end{array}\right|
$$

is different from zero, and the equations $\Omega_{\mu}=0$ are satisfied at $x=x_{1}$. This can always be done, since, as proved in $\S 3$, the functions $\lambda(x)$ are never simultaneously zero and the determinant $\left|\psi_{a \beta}\right|$ is different from zero. The corresponding solutions $u^{(\sigma)}, \rho^{(\sigma)}$ of the equations (29) form the system required.

Any system having the properties of the theorem will make the determinant (33) different from zero. For, consider such a system, not necessarily the one just determined. If (33) is zero, constants $c_{\sigma}, c$ can be found satisfying the linear equations whose coefficients are the values assumed at $x=x_{1}$ by the functions in the rows of (33). The solution

$$
\eta=\sum_{\sigma} c_{\sigma} u^{(\sigma)}, \quad \mu=\sum_{\sigma} c_{\sigma} \rho^{(\sigma)}+c \lambda
$$

would have $\eta_{i}\left(x_{1}\right)=\eta_{r}^{\prime}\left(x_{1}\right)=\mu_{\alpha}\left(x_{1}\right)=0$ for every $i, r, \alpha$, since the equations $\Omega_{\mu}=0$ are satisfied and the determinant $\left|\psi_{a \beta}\right|$ is different from zero, it would also have $\eta_{a}^{\prime}\left(x_{1}\right)=0$. Being a linear combination of solutions of the equations (28), it is evidently a solution of (29). Hence $\eta(x) \equiv \mu(x) \equiv 0$.

Now, if $\eta, \mu$ constitute an arbitrary solution of the Jacobi equation, since the determinant (33) is different from zero, it is possible to determine $c_{\sigma}, c$ so as to satisfy the linear equations

$$
\begin{gathered}
\eta_{i}\left(x_{1}\right)=\sum_{\sigma} c_{\sigma} u_{i}^{(\sigma)}\left(x_{1}\right), \quad \eta_{r}^{\prime}\left(x_{1}\right)=\sum_{\sigma} c_{\sigma} u_{r}^{(\sigma) \prime}\left(x_{1}\right), \\
\mu_{a}\left(x_{1}\right)=\sum_{\sigma} c_{\sigma} \rho^{(\sigma)}\left(x_{1}\right)+c \lambda_{a}\left(x_{1}\right) .
\end{gathered}
$$

The solution $\eta=\eta(x)-\sum_{\sigma} c_{\sigma} u^{(\sigma)}(x), \bar{\mu}=\mu(x)-\sum_{\sigma} c_{\sigma} \rho^{(\sigma)}-c \lambda$ of equations (29) has $\bar{\eta}_{i}\left(x_{1}\right)=\eta_{r}^{\prime}\left(x_{1}\right)=\bar{\mu}\left(x_{1}\right)=0$ and, since it satisfies $\Omega_{\mu}=0$ has $\eta_{a}^{\prime}\left(x_{1}\right)=0$. Then $\bar{\eta}(x) \equiv \vec{\mu}(x) \equiv 0$, and

$$
\eta=\sum_{\sigma} c_{\sigma} u^{(\sigma)}(x), \quad \mu=\sum_{\sigma} c_{\sigma} \rho^{(\sigma)}(x)+c \lambda(x) .
$$

This completes the proof of the theorem.

THEOREM 4. If $u^{(\sigma)}, \rho^{(\sigma)}$ are $2 n+1$ sets of solutions of the Jacobi equations satisfying the hypotheses of Theorem 3 , the points conjugate to $P_{1}$ on the extremal $E$ are defined by the zeros of the determinant

$$
D\left(x_{1}, x\right)=\left|\begin{array}{l}
u_{i}^{(\sigma)}\left(x_{1}\right) \\
u_{p}^{(\sigma)}(x)
\end{array}\right|
$$

where $\sigma$ varies in the rows and $i$ and $p$ in the columns. 
Every such zero is a conjugate point. For

$$
u=\sum_{\sigma} c_{\sigma} u^{(\sigma)}, \quad \rho=\sum_{\sigma} c_{\sigma} \rho^{(\sigma)}
$$

are a solution of the Jacobi equations and, if $D\left(x_{1}, x_{3}\right)=0$, there must exist $c_{\sigma}$ not all zero such that

$$
u_{i}\left(x_{1}\right)=\sum_{\sigma} c_{\sigma} u_{i}^{(\sigma)}\left(x_{1}\right)=0, \quad u_{p}\left(x_{3}\right)=\sum_{\sigma} c_{\sigma} u_{p}^{(\sigma)}\left(x_{3}\right)=0 .
$$

The sum $F_{y^{\prime}} u$ is a constant, as shown in $\S 3$; the arc $E$ being normal on $x_{1} x_{3}, F_{y_{0}^{\prime}}\left(x_{3}\right)$ is different from zero, so the value $u_{0}\left(x_{3}\right)$ is also zero. The functions $u$ cannot be identically zero, since from Theorem 2 and the corollary to Theorem 1, the function $\rho$ would then be of the form $\rho \equiv c \lambda$ and the solutions $u^{(\sigma)}, \rho^{(\sigma)}$ would not be independent in the sense of Theorem 3. By the definition of a conjugate point, $x_{3}$ defines a point conjugate to $P_{1}$.

On the other hand, since every solution of (28) is expressible as in Theorem 3 , every $x_{3}$ corresponding to a conjugate point is a zero of $D\left(x_{1}, x\right)$.

The conjugate point may also be determined from the general solution of the Euler differential equations of the original Mayer Problem. The lemma and theorem following establish such a method as a direct consequence of the results just obtained.

Lemma. If $y=e(x, a), \lambda=\lambda(x, a)$ form a one-parameter family of solutions of the Euler equations containing the minimizing arc $E$ for $a=0$, then the functions $u=e_{a}(x, 0), \lambda=\lambda_{a}(x, 0)$ are a system of solutions of the $J a c o b i$ equations for $E$.

This is proved, as usual, by differentiating with respect to $a$ the identities in $x$ obtained by substituting $y=e(x, a), \lambda=\lambda(x, a)$ in the Euler equations (13).

TheOREM 5. Consider a $(2 n+1)$-parameter family of extremals

$$
y=e(x, a), \quad \lambda=\lambda(x, a),
$$

where $a$ is the $(2 n+1)$-partite parameter $\left(a_{1}, \cdots, a_{2 n+1}\right)$. Suppose this family contains $E$ for $a=0$, and that the functions

$$
u^{(\sigma)}(x)=\left[\frac{\partial e}{\partial a_{\sigma}}\right]^{a=0}, \quad \rho^{(\sigma)}(x)=\left[\frac{\partial \lambda}{\partial a}\right]^{a=0}
$$

are linearly independent in the sense of Theorem 3. Then the points conjugcte to $P_{1}$ on $E$ are defined by the zeros of the determinant

$$
D\left(x_{1}, x\right)=\left|\begin{array}{l}
u_{i}^{(\sigma)}(x) \\
u_{p}^{(\sigma)}(x)
\end{array}\right| .
$$

This follows at once from the lemma and from Theorem 4. 
The existence of such a family may be shown as follows. The Euler equations (13) are equivalent to the system

$$
F_{y}-\frac{d}{d x} F_{y^{\prime}}=0 ; \quad \frac{d \phi}{d x}=0
$$

with the initial conditions $\phi=0$ at $x=x_{1}$. These equations are of the second order in the functions $y$, of the first order in the functions $\lambda$, and linear in the derivatives $y^{\prime \prime}, \lambda^{\prime}$. The determinant of the coefficients of $y^{\prime \prime}, \lambda^{\prime}$ is the determinant $R\left(x, y, y^{\prime}, \lambda\right)$, which is different from zero along $E$. Let $l_{\alpha}(x)$ be the set of multipliers $\lambda$ for $E$. At least one of these multipliers is different from zero at $x=x_{1}$. Suppose $l_{0}\left(x_{1}\right) \neq 0$ and alter the functions by a constant multiplier so that $l_{0}\left(x_{1}\right)$ is equal to unity. Define the initial values of the functions $y, \lambda$ in terms of $a_{1}, \cdots, a_{2 n+1}$ by the equations

$$
\begin{aligned}
& y_{i}\left(x_{1}\right)=e_{i}\left(x_{1}\right)+a_{i}, \quad y_{r}^{\prime}\left(x_{1}\right)=e_{r}^{\prime}\left(x_{1}\right)+a_{n+1+r}, \\
& \lambda_{0}\left(x_{1}\right)=1, \lambda\left(x_{1}\right)=l\left(x_{1}\right)+a_{2 n-m+1}, \cdots, \lambda_{m}\left(x_{1}\right)=l_{m}\left(x_{1}\right)+a_{2 n+1} .
\end{aligned}
$$

The initial values $y_{a}\left(x_{1}\right)$ are then determined in terms of $a_{1}, \cdots, a_{2 n+1}$ by the equations $\phi\left[x, y\left(x_{1}\right), y^{\prime}\left(x_{1}\right)\right]=0$ : The usual existence theorems for differential equations establish the existence of a family of solutions (34) satisfying the initial conditions (36). From the latter it appears that the determinant (33) used in the proof of Theorem 3 is equal to unity and the functions (35) are, therefore, independent in the sense of Theorem 3.

Randolfh-Macon Woman's College. 